\title{
Gas-Oil Relative Permeability and Residual Oil Saturation as Related to Displacement Instability and Dimensionless Numbers
}

\author{
B. Rostami 1, 2, R. Kharrat ${ }^{*}$, C. Ghotbi ${ }^{2}$ and S.H. Tabatabaie ${ }^{3}$ \\ 1 Petroleum University of Technology, Tehran Research Center, Tehran 1453953111 - Iran \\ 2 Sharif University of Technology, Azadi Ave., PO Box 11365-8639, Tehran - Iran \\ 3 University of Calgary, Calgary, Alberta, Canada \\ e-mail: rostami@che.sharif.ir - kharrat@put.ac.ir - ghotbi@sharif.edu - h.tabatabaie@ucalgary.ca \\ *Corresponding author
}

\begin{abstract}
Résumé - Étude de la perméabilité relative gaz-huile et de la saturation en huile résiduelle dans le cas d'une instabilité de déplacement et des nombres sans dimension s'y rapportant - Des expériences de déplacement gaz-huile ont été réalisées sur des modèles mis à l'échelle de carottes de grande longueur en faisant varier les propriétés pétrophysiques et les conditions d'écoulement. Pour ces expériences, les forces en présence, capillaires, gravitaires et visqueuses, sont comparables. Le seuil de stabilité est déterminé à partir de l'historique de production et de l'analyse d'images. Les résultats des expériences sont comparables aux conclusions de la théorie de la percolation en gradient. On étudie ensuite l'effet de l'instabilité du front de déplacement sur la perméabilité relative et la saturation résiduelle. Les perméabilités relatives déterminées par des approches analytiques et numériques indiquent qu'une plus grande vitesse de déplacement engendre une plus grande perméabilité relative au gaz et une plus faible perméabilité relative à l'huile. Les résultats indiquent que la saturation en huile à la fin est très supérieure lorsque la vitesse de déplacement se situe au-dessus du critère de stabilité. Les caractéristiques du déplacement, notamment la saturation moyenne en huile en fin de déplacement, sont ensuite décrites à l'aide de groupements sans dimension expressément le nombre de Bond et le nombre capillaire. La saturation en huile en fin de déplacement déterminée expérimentalement s'exprime respectivement par une relation directe avec le nombre capillaire et inverse avec le nombre de Bond. En conséquence, un groupement sans dimension combiné a été proposé afin de généraliser l'estimation de la saturation en fin de déplacement et résiduelle en huile dans la limite des nombres sans dimension étudiés ici.
\end{abstract}

\footnotetext{
Abstract - Gas-Oil Relative Permeability and Residual Oil Saturation as Related to Displacement Instability and Dimensionless Numbers - Displacement experiments of the gas-oil system are performed on long core scale models by varying the petrophysical properties and flowing conditions. Experiments are conducted in situations where capillary, gravity and viscous forces are comparable. From oil production history and picture analysis, the threshold for the stability is determined. The experimental findings are comparable to the results of a gradient percolation theory. The effect of destabilized front velocity on relative permeability and residual saturation is investigated. The relative permeabilities
} 
determined by using analytical and numerical approaches indicate that higher displacement velocity leads to a higher gas relative permeability and lower oil relative permeability. The remaining oil saturation is found to be much higher for displacement velocity above the stabilized criterion. Displacement morphology including the average remaining oil saturation is then described using dimensionless groups expressed as Bond and capillary number. Experimentally determined remaining oil saturation shows a direct and inverse relation to the capillary and Bond number respectively. Hence, a combined dimensionless group has been proposed to generalize the estimation of remaining and residual oil saturations under the range of dimensionless numbers studied here.

\section{NOMENCLATURE}

$f \quad$ Fractional flow function

$g \quad$ Gravitational constant $\left(\mathrm{m} / \mathrm{s}^{2}\right)$

$k$ Absolute permeability $(\mathrm{mD})$

$k_{r o} \quad$ Oil relative permeability

$k_{r o}^{o} \quad$ Curve-fitting parameter

$L \quad$ Length of porous medium (cm)

$n \quad$ Relative permeability exponent

$N_{B} \quad$ Bond number, ratio of gravity to capillary forces

$N_{B}^{*} \quad$ Generalized Bond number

$N_{c} \quad$ Capillary number, ratio of viscous to capillary forces

$N_{c, \text { end }}$ Capillary end-effect number

$N_{c g} \quad$ Capillary to gravity forces

$N_{c o} \quad$ Combined dimensionless number

$N_{g v} \quad$ Gravity number, ratio of gravity to viscous forces

$N_{p} \quad$ Produced oil (fraction)

$p \quad$ Pressure (Psi)

$p_{c} \quad$ Capillary pressure (Psi)

$p_{t h} \quad$ Threshold capillary pressure (Psi)

$q_{i} \quad$ Injection rate (cc/min)

$s \quad$ Saturation

$s_{\text {oDe }} \quad$ Outlet normalized oil saturation

$s_{\text {org }} \quad$ Residual oil saturation

$s_{\text {rem }}$ Remaining oil saturation

$t \quad$ Time, $\min$

$u \quad$ Velocity $(\mathrm{cm} / \mathrm{min})$

$v \quad$ Velocity $(\mathrm{cm} / \mathrm{min})$

$v_{c} \quad$ Critical gravity drainage rate $(\mathrm{cm} / \mathrm{min})$

$w \quad$ Invasion front width

Z Length (cm)

\section{Greeks}

$\varphi \quad$ Porosity

$\sigma \quad$ Interfacial tension, $(\mathrm{mN} / \mathrm{m})$

$\Delta \rho \quad$ Density difference of gas-oil $\left(\mathrm{kg} / \mathrm{m}^{3}\right)$

$\Delta \mu \quad$ Viscosity difference of gas-oil (cP)

$\Delta P \quad$ Imposed pressure differential (Psi)

$\mu \quad$ Viscosity (cP)

$\gamma \quad$ Specific gravity

\section{Subscripts}

D Dimensionless

$g \quad$ Gas

$o \quad$ Oil

w Water

\section{INTRODUCTION}

Fluid displacement in porous medium is of interest for many important natural and engineering processes. Immiscible displacement of fluids in porous medium occurs in a wide range of flow regimes that reflect the interactions between capillary, gravity and viscous forces. Experiments performed on artificial porous medium (Fourar et al., 1993; Avraam and Payatakes, 1995a; Persoff and Pruess, 1995), as well as pore network simulations (Blunt and King, 1993; Goode and Ramakrishnan, 1993; Vizika et al., 1994; Constantinides and Payatakes, 1996; Aker et al., 1998; Hughes and Blunt, 2000; Singh and Mohanty, 2003; Tsakiroglou et al., 2005; Theodoropoulou et al., 2005), have revealed that the transient and steady-state two phase flow patterns depend not only on the specific characteristics of the pore space morphology, but also on a variety of dimensionless parameters such as the capillary number, viscosity ratio, wettability. The relative permeability functions of porous medium are strongly correlated with the flow regimes and are strongly dependent on the capillary number and viscosity ratio (Avraam and Payatakes, 1995a; Singh et al., 2001; Tsakiroglou et al., 2003). Moreover, invasion percolation and gradient percolation models have also been employed to interpret quantitatively the growth of stabilized and destabilized fronts under the interactions of gravity, capillarity, and viscous forces (Wilkinson, 1986; Gouyet et al., 1988; Cieplak and Robbins, 1990; Ferer et al., 1995; Zhang and Liu, 1998; Xu et al., 1998; Meheust et al., 2002; Ferer et al., 2003; Lovell et al., 2005).

For gas-oil gravity drainage with high viscosity contrast, where the interplay between capillary, gravity and viscous forces controls the invasion front, interaction of transport functions and prevailing forces becomes important. A number of investigations are focused on key factors that affect front morphology. The interest in displacement front behavior is motivated by potential impact on fluid phase distributions with associated effects on macroscopic transport properties 
such as relative permeability and capillary pressure (Or, 2008). A criterion based on gravity invasion percolation theory has been proposed by Meheust et al. (2002) and Lovell et al. (2005). They used the percolation theory to predict the vertical extension of the displacement of the invasion fronts. They used generalized Bond number and showed invasion instability when this number approaches to zero. A simple criterion based on dimensionless numbers also proposed by Raats (1973) and expanded by Philip (1975) suggests that flow instability sets in whenever the pressure gradient at the wetting front opposes the direction of flow. Or (2008) described the displacement fluid front morphology using dimensionless numbers and proposed a limitation to define conditions for onset of unstable and intermittent flows leading to enhanced liquid and gas entrapment.

A numbers of investigations also have been carried out to correlate the gas-oil relative permeability and residual saturation with different flow regimes and dimensionless numbers. Skauge et al. (1997) conducted series of centrifuge and gasflood experiments and showed more dispersed gas front and lower recovery efficiency at higher gas flow rate. Their parameter estimation technique for determination of relative permeabilities also indicated higher gas relative permeability at higher rate and slightly changes in oil relative permeability by flow rate. The experimental and theoretical analysis of Edwards et al. (1999) showed differences between recoveries by process where gravity control the flood front and by those where the gravitational forces play less significant role in ultimate fluid distribution. They also described the effect of flow regimes on end point saturations and gas-oil relative permeabilities. Singh et al. (2001) compared the viscous and gravity dominated relative permeabilities and showed that separate relative permeability and residual saturation values need to be plugged into a reservoir simulator upon the relative magnitude of the viscous to the gravity forces.

Usually, an estimate of relative permeability and residual oil saturation is obtained by carrying out displacement experiments. The laboratory experiments for estimating relative permeability include centrifuge displacements, unsteady state gas flood and steady state measurements. Hirasaki et al. (1995) believed that for centrifuge method limitations include loss of information on the low saturation region that can not be gained from production data at low mobility ratio. Ali (1997) stated that "concerns remain regarding the replacement of centrifugal forces for unsteady state displacement process that are rate dependent". Firoozabadi and Aziz (1988) mentioned that steady state methods offer disadvantages, especially in the case of low permeable rocks where it is laborious to reach multiple steady states. Akin and Kovscek (1999) showed that the use of the (unsteady) JBN technique leads to inaccurate of relative permeability in some cases. Mohanty and Miller (1991) used the capillary end-effect dimensionless number, defined by Equation (1), and stated that for $N_{c, \text { end }}>0.02$, as $N_{c, \text { end }}$ increases the derived relative permeabilities decrease in drainage experiments. This indicates that for $N_{c, \text { end }}>0.02$, the capillary end-effect is important and needs to be taken into account while interpreting the lab scale gas flood experiments. Hagoort also introduced a criterion for validity of his method. He used the capillary to gravity number and showed that for $N_{c g}>10^{-3}$ the capillary effect is important and needs to be taken into account. Saeedi (2007) performed a large number of centrifuge experiments and numerical investigation and stated that if the capillary to gravity number, $N_{c g}$, is maintained in order of $10^{-2}$ or smaller, the capillary end-effect becomes negligible.

$$
N_{c, \text { end }}=\left(p_{t h} / \Delta p\right)
$$

There are also methods that infer relative permeability by history matching observable parameters such as fluid production, pressure drop and saturation history (Schember and Kovscek, 2003). The limitation imposed on the description of the actual shape of relative permeability curve causes a bios error and a variance error (Watson et al., 1996).

In this paper, experimental results were obtained in vertical long core and synthetic porous medium. In each medium, we study the transition from flow configurations where the interface is stable with respect to the stabilized front velocity to flow configurations where the viscous fingering occurs. In order to check under which circumstance the stabilization occurs, the evolution of the front instability is investigated and compared to the gravity invasion percolation theory. Then, the influence of displacement instability on relative permeabilities and residual saturations is investigated using analytical and numerical methods. The oil relative permeability is obtained by unsteady state displacement data using an analytical approach with no need for pressure data. Then, the gas relative permeability is calculated using the numerical history matching technique. We also review the impact of proper dimensionless numbers on remaining oil saturation and generalize this interpretation by introducing a combined group to identify a flow behavior during all experiments.

\section{EXPERIMENTAL}

\subsection{Porous Medium and Fluids}

The forced gravity drainage experiments have been performed in synthetic porous medium (bead-pack and sand-pack with average grain size of $150 \mu \mathrm{m}$ and $100 \mu \mathrm{m}$ respectively). The average porosity was around $37 \%$ and the absolute permeability ranged between 3 and 20 Darcy. Although these homogenous test sections are a simplistic representation of the pore space, they can accurately represent the main flow characteristics of reservoir rocks; especially when the ranges of dimensionless numbers are located in the field range. The porous medium characteristics for each experiment are given in Table 1. 
TABLE 1

The key parameters for the selected pairs of forced gravity drainage experiments

\begin{tabular}{c|c|c|c|c|c|c|c|c}
\hline Porous medium & Exp. number & $\varphi$ (fraction) & $A\left(\mathrm{~cm}^{2}\right)$ & $L(\mathrm{~cm})$ & $K(\mathrm{mD})$ & $q_{i}(\mathrm{cc} / \mathrm{min})$ & $N_{c}\left(10^{-5}\right)$ & $N_{B}\left(10^{-5}\right)$ \\
\hline \multirow{5}{*}{ Bead pack } & a1 & 0.38 & 7.06 & 120 & 18500 & 3.1 & 0.31 \\
(Visual model) & a2 & 0.375 & 7.06 & 120 & 17800 & 8.0 & 0.81 \\
& a3 & 0.378 & 7.06 & 120 & 17000 & 16.5 & 1.65 \\
& b1 & 0.365 & 7.06 & 120 & 8800 & 5 & 1.72 \\
& b2 & 0.37 & 7.06 & 120 & 9000 & 10 & 1.63 \\
Sand pack & b3 & 0.375 & 7.06 & 120 & 8500 & 16 & 1.0 & 0.87 \\
& c1 & 0.375 & 12.56 & 60 & 3700 & 2 & 0.88 \\
& c2 & 0.38 & 12.56 & 60 & 3500 & 5.5 & 0.11 & 0.31 \\
\hline
\end{tabular}

The fluids are carefully prepared to avoid any impurity that would alter their physical properties. The gas phase is normally Nitrogen and synthetic oil (n-decane) is selected as an oil phase. Their physical properties are measured using standard techniques and given in Table 2.

\section{TABLE 2}

Fluid properties@ standard conditions

\begin{tabular}{c|c|c|c|c}
\hline $\begin{array}{c}\text { Oil density } \\
\left(\mathrm{kg} / \mathrm{m}^{3}\right)\end{array}$ & $\begin{array}{c}\text { Oil viscosity } \\
(\mathrm{cP})\end{array}$ & $\begin{array}{c}\text { Gas density } \\
\left(\mathrm{kg} / \mathrm{m}^{3}\right)\end{array}$ & $\begin{array}{c}\text { Gas viscosity } \\
(\mathrm{cP})\end{array}$ & $\begin{array}{c}\text { IFT } \\
(\mathrm{mN} / \mathrm{m})\end{array}$ \\
\hline 740 & 0.85 & 1 & 0.018 & 20 \\
\hline
\end{tabular}

\subsection{Apparatus and Procedure}

The schematic drawing of the equipment used in the laboratory is shown in Figure 1. The complete experimental setup includes the long cylindrical visual model and the high pressure core holder, fluid injection system, gas injection system, differential gauge pressure, effluent collection system consists of two phase separator and digital balance, gas cylinder, digital camera and data acquisition system.

A cylindrical core holder made of $4 \mathrm{~cm}$ (outside diameter) Plexiglass column was used in this study. Plexiglass is easy to design and a relatively more transparent material for visual observations. So that, any movement of the fluids can be observed. Another core holder made of Aluminum was used for sand-pack experiments. This model was designed to be at least $60 \mathrm{~cm}$ in length, however the overall length was $85 \mathrm{~cm}$; that is, an additional $10 \mathrm{~cm}$ on the inlet end and $15 \mathrm{~cm}$ on the outlet end were left to minimize the inlet and outlet effect. The inlet and outlet valves were inserted in top and bottom caps of the model with $1 / 8$ " line. The differential gauge pressure was fitted to the inlet port. Since the outlet end is released to the atmospheric pressure, the gauge pressure at the inlet gives the differential pressure during the displacements. Both models were mounted on a metallic base with a gear that allowed rotating and positioning the model at different directions and angles. High pressure pumps were used to

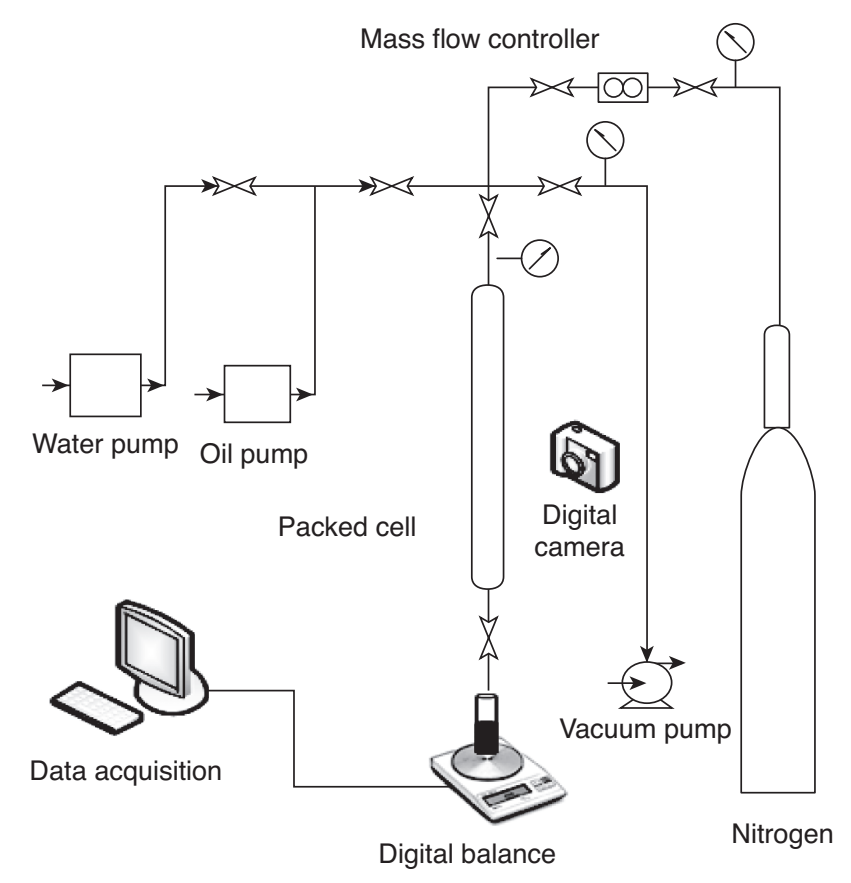

Figure 1

Schematic drawing of the laboratory experimental set up.

inject liquids and a Mass Flow Controller (MFC) was used for measuring and controlling flow rate of gases. Two-phase separator located at the production end is used for collecting fluids. Because of the error introduced due to improper visual readings of the fluid meniscus, the commonly used method of measuring volumes of the effluents was not used. Instead, an incremental weight measurement method using a digital computer controlled balance was used to measure the effluent production data. Moreover, the displacement structures are recorded at regular intervals during the experiments by digital camera.

The experiments were conducted under ambient laboratory temperature and the outlet face of the model was open to atmospheric pressure. For each experiment, the apparatus 
was assembled, the model was placed in vertical gravity stable position and a leak test was performed by applying gas pressure and verifying that pressure was maintained for a period of time. Before starting experiment, the sintered pack was first vacuumed until desired pressure, and then fully saturated with the oil sample. The porosity and absolute permeability was then measured. Nitrogen was injected through the mass flow controller under specific rates. Various recording were made during the experiments. Pressure is recorded at the inlet. Oil production was monitored continuously using the computer controlled system at the outlet for further analysis. The displacement structures are also taken at regular intervals during the experiments. These raw pictures were filtered in order to clearly separate the wetting from the nonwetting phase and to extract the invasion front. Successive steps of this filtering process are presented in Figure 2.

\section{MATHEMATICAL MODELING}

In this section, the analytical method and numerical model are described. The proposed analytical method is used for determination of the relative permeability to oil whereas the mathematical experimentation indicated that the oil production history is independent of gas relative permeability when the front velocity is stable (results are not shown here). For high velocity experiments when the relative permeability to gas is important, after determination of oil relative permeability by analytical method, the gas relative permeability is obtained using history matching technique. In all cases, the gas and oil relative permeability curves obtained from the analytical and numerical procedures are used as an input for numerical model and the agreement between experimental results and numerical data is compared.

\subsection{Analytical Method}

The basis of analytical method used here is that of Hagoort (1980). Hagoort introduced a backward methodology for determination of oil relative permeability from centrifugal data. He assumed that the capillary pressure is negligible and the gas phase has an infinite mobility. Hence, Hagoort's method could only give the relative permeability to oil. However, in forced gravity drainage flow when the displacement velocity is exceeded than the stabilized criterion, defined in Section 4.1, the viscous force can have a large effect on ultimate fluid distribution. In this circumstance, the gas relative permeability becomes important and needs to be taken into account. In this study, the history matching technique for estimating this function is suggested.

\subsubsection{Formulation}

Hagoort simplified the Buckley-Leveret solution by assuming negligible capillary pressure, infinite gas mobility and a Corey type oil relative permeability function.

The general equation for incompressible fractional flow of oil phase in presence of the viscous, capillary and gravity forces in dimensionless form is presented by Equation (2). Definitions of dimensionless parameters used in this equation are listed in Equation (3).

$$
\begin{gathered}
\frac{\partial S_{o D}}{\partial t_{D}}+\frac{\partial f_{o D}}{\partial z_{D}}=0 \\
S_{o D}=\frac{S_{0}-S_{o r g}}{1-S_{o r g}-S_{i w}}, t_{D}=\frac{\Delta \rho_{o g} g k}{\mu_{o} \varphi\left(1-S_{o r g}-S_{i w}\right) L}, Z_{D}=\frac{z}{L}
\end{gathered}
$$

$f_{O D}$ is the normalized fractional flow function which is defined in the following. Details of $f_{o D}$ derivation can be found in Hagoort (1980).

$$
f_{o D}=\frac{\frac{1}{N_{g v}} \frac{\mu_{g} k_{r o}}{k_{r g} \mu_{o}}+k_{r o}+N_{c g} k_{r o} \frac{\partial J}{\partial S_{o D}} \frac{\partial S_{o D}}{\partial z_{D}}}{1+\frac{\mu_{g} k_{r o}}{k_{r g} \mu_{o}}}
$$

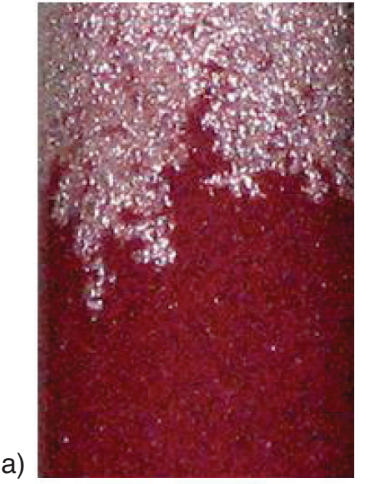

b)
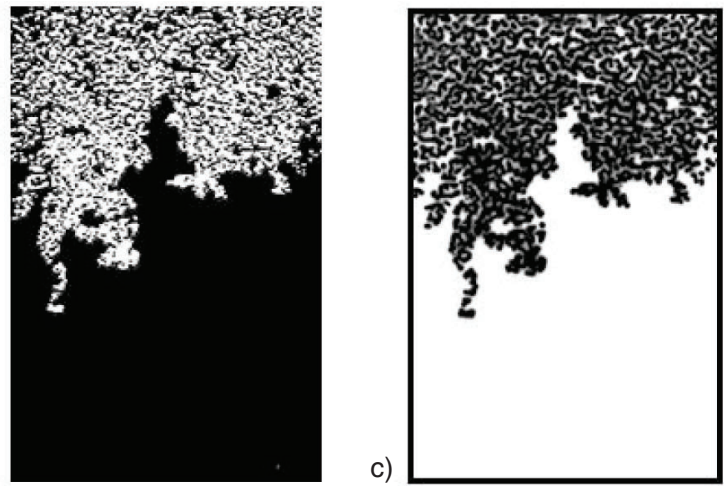

Figure 2

Image filtering process: a) the raw image; b) black and white image; c) image in reverse color. 
where:

$$
N_{g v}=\frac{\Delta \rho_{o g} g k}{\mu_{o} u}
$$

is the ratio of the gravity to viscous forces,

$$
N_{c g}=\frac{\sigma \sqrt{\varphi / k}}{\Delta \rho_{o g} g l}
$$

is the ratio of the capillary to gravity forces, and

$$
J\left(S_{o D}\right)=\frac{\sigma \sqrt{\varphi / k}}{p_{c}}
$$

is the capillary pressure relationship.

As a result of Hagoort's assumptions, negligible capillary pressure and infinite gas mobility, the fractional flow function depends only on the reduced saturation.

$$
f_{o D}=k_{r o}\left(S_{o D}\right)
$$

and the fractional flow equation becomes:

$$
\frac{\partial S_{o D}}{\partial t_{D}}+\frac{\partial k_{r o}}{\partial S_{o D}} \frac{\partial S_{o D}}{\partial z_{D}}=0
$$

This Equation has been solved by the method of characterization like the Buckley-Leverett solution.

$$
z_{D}\left(S_{o D}\right)=\frac{\partial k_{r o}}{\partial S_{o D}} t_{D}\left(S_{o D}\right)
$$

Once the saturation profiles are known, the oil production versus time can be calculated readily. Before gas breakthrough the oil production must equal to the amount of gas injected.

$$
N_{p}=\frac{\int_{0}^{t} q_{t} d t}{P V \times L}=\frac{t_{D}}{N_{g v}}
$$

In the absence of capillary forces, the oil production after gas breakthrough is as follow:

$$
N_{p}=1-\int_{0}^{S_{o D}}\left(1-z_{S_{o D}}\right) d S_{o D}=1-S_{o D e}+k_{r o}\left(S_{o D e}\right) t_{D}
$$

where $z_{S_{O D}}$ is the vertical distance at the specific saturation, and $S_{o D e}$ is the outflow normalized oil saturation which is defined by Equation (11). When the relative permeability follows the Corey type function, $k_{r o}=k_{r o}^{o} S_{o D}^{n}$, Equation (9) becomes:

$$
N_{p}=1-S_{o D e}+k_{r o}^{\circ} S_{o D}^{n} t_{D}
$$

At the outflow end, $z_{D}=1$, Equation (7) becomes:

$$
1=\frac{\partial k_{r o}}{\partial S_{o D}} t_{D}\left(S_{o D}\right)
$$

After substitution of the Corey type oil relative permeability, it gives the outflow normalized saturation:

$$
S_{o D e}=\left(\frac{1}{n k_{r o}^{\circ} t_{D}}\right)^{\frac{1}{n-1}}
$$

Substituting Equation (11) in Equation (10) gives:

$$
N_{p}=1-\left(1-\frac{1}{n}\right)\left(\frac{1}{n k_{r o}^{o} t_{D}}\right)^{\frac{1}{n-1}}
$$

Note that Equation (12) is the forward solution for $N_{p}$, the backward solution requires the use of $N_{p}$ versus $t_{D}$ data in order to find $k_{r o}\left(S_{o D e}\right)$.

Based in Equation (12), a plot of $1-N_{p}$ versus $t_{D}$ on a log-log paper should be a straight line. Then the following equation can be used to calculate the value of Corey exponent from the straight line.

$$
\frac{d \ln \left(1-N_{p}\right)}{d \ln t_{D}}=-\left(\frac{1}{n-1}\right)
$$

For $t_{D}=1$, we then can obtain $k_{r o}^{o}$ from Equation (12):

$$
k_{r o}^{o}=\frac{1}{n}\left(\frac{n-1}{n\left(1-N_{p}\right)}\right)^{n-1}
$$

Hagoort used $k_{r o}^{o}$ as a curve fitting parameter and stated that this need not have any significance with respect to the endpoint of the oil relative permeability curve.

Numerical experimentations showed that at the high displacement velocity tests, oil production at early time is affected by the gas mobility and the production data exactly after breakthrough time could not fully represent the oil mobility. Here, it suggests that the straight line plots some times after gas breakthrough to exclude the effect of gas mobility on oil production data. The numerical and experimental results given in this paper demonstrate that this modification could be easily applied graphically and the oil relative permeability exponent is estimated from the transient production data.

\subsubsection{Limitation}

The proposed calculation method is neglected the capillary term, as well as the other routine methods such as JBN, for development or simplified interpretation technique. However, the displacements are often affected by the capillary end-effect. In this situation, the obtained relative permeability curves should be corrected.

In this study, history matching technique based on parameter estimation is used to calculate the corrected functions when the capillary end-effect needs to be considered. Details of this method are explained in Section 2.2.3. When the obtained curves were used in the forward numerical model, 
the production results from this numerical simulation could satisfactory mach the experimental data. Although this methodology may still be non-unique, the obtained results could be reasonable candidate of real curves.

\subsubsection{A Sample Relative Permeability Calculation}

In this section, the step by step treatment of data for calculation of gas/oil relative permeability for a typical case is presented.

The oil production data was used along with the backwardanalytical model to find a Corey type oil relative permeability and History matching code for determination of gas relative permeability. Figure 3 shows complete steps of such backward/forward model for determination of two phase gas/oil relative permeabilities. For determination of residual oil saturation the experiments were continued until no more oil produced. The graphical method presented by Jones and
Roszelle (1978) can be used to predict the final oil volume. This can help to ensure that the experimentally reported final oil production does actually represent the oil production at a very large throughput. The production data from experiments "bl" is presented in Figure 3a. The breakthrough time can found from the inflection point of the production data versus time. Previously, it is explained that the production data after breakthrough is used for relative permeability measurement. Figure $3 \mathrm{~b}$ shows a straight line on the plot of $1-N_{p}$ versus $t_{D}$ on log-log scale. The slope of the straight line plotted in this figure can be used for estimation of Corey oil relative permeability exponent. Result suggests $n=3.03, S_{o D e}=0.43$ and $k_{r o}^{o}=1.78$ (Fig. 3c). Since the capillary end-effect was not significant in this experiment, the correction procedure has not been employed. For estimating the gas relative permeability, the calculated oil relative permeability from the
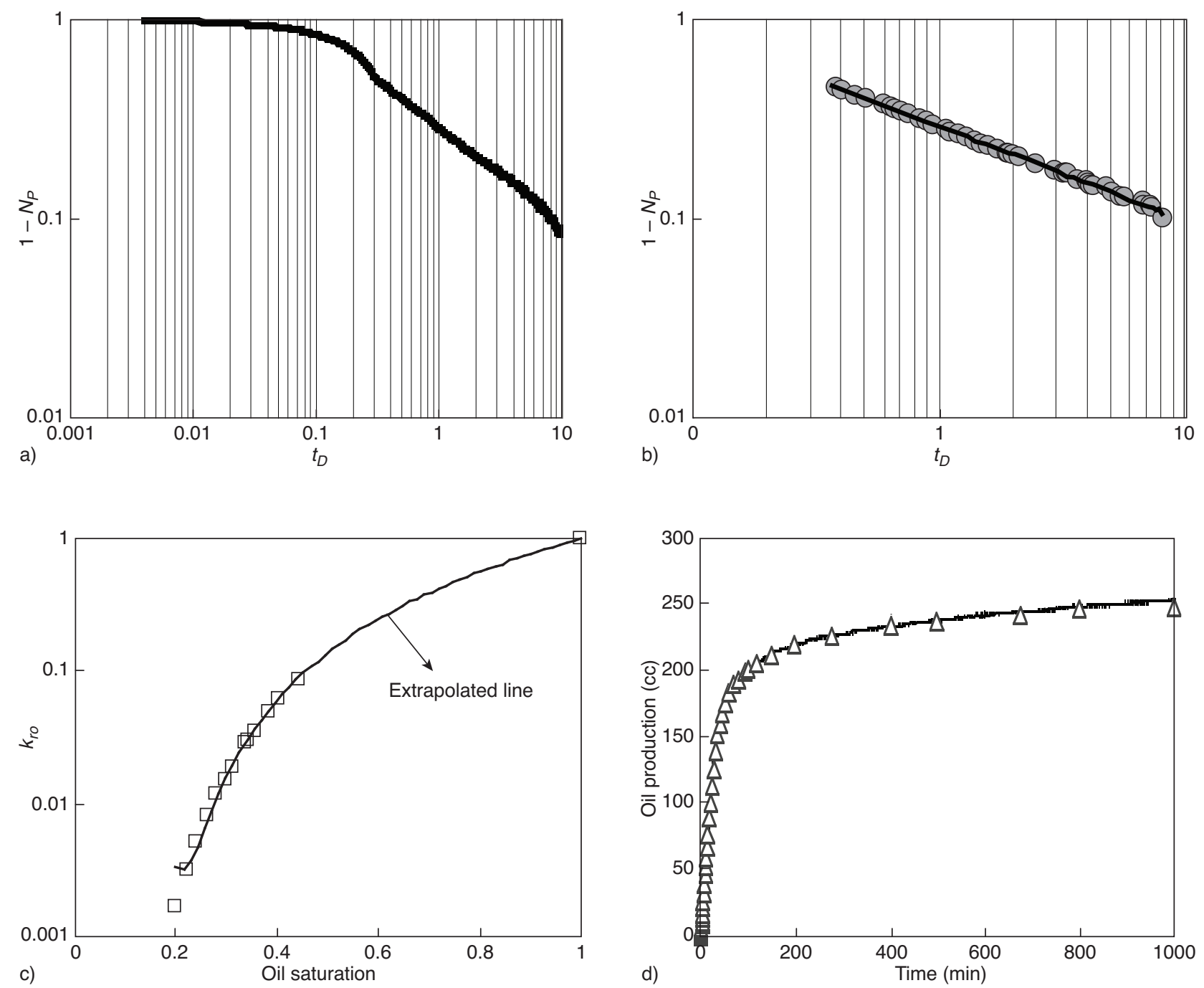

Figure 3

A sample relative permeability calculations for a typical gravity dominated experiment. 
analytical method is used in forward numerical model and a synthetic, but appropriate, Corey type gas relative permeability is inserted into the numerical model as a first guess. The other physical and fluid parameters are known from the experiments. Then, based on history matching technique the agreement between numerically produced oil recovery and experimental data has been checked. The gas Corey exponent and end-point values were changed until reasonable match obtained. Finally, the obtained relative permeability functions are used along with the numerical model to predict the experimentally oil production. In part (d) of Figure 3, experimentally production data is compared to simulated production profile using the analytically determined oil relative permeability and history matched relative permeability to gas for a typical test.

\subsection{Numerical Model}

The one-dimensional (1-D) flow of two incompressible fluids in a homogenous and isotropic porous medium is written by the mass (continuity equation) and momentum (Darcy equation) balances. In this model, gas enters from the top of an oil saturated column and oil is produced from the bottom. The oil drainage is simulated and predicted by accounting for the gravity, capillary and viscous forces.

\subsubsection{Formulation}

For the case of vertical, downward displacement of oil by gas, the oil and gas velocities are given by the Darcy's law:

$$
\begin{aligned}
& u_{o}=-\frac{k k_{r_{o}}}{\mu_{o}}\left(\frac{\partial P_{o}}{\partial x}-\gamma_{o} \frac{\partial z}{\partial x}\right) \\
& u_{o}=-\frac{k k_{r_{0}}}{\mu_{o}}\left(\frac{\partial P_{o}}{\partial x}-\gamma_{o} \frac{\partial z}{\partial x}\right)
\end{aligned}
$$

The saturation distribution of each phase in the porous medium during multiphase flow is described by the continuity equation, which is the mathematical form of the mass conservation principle.

$$
\begin{gathered}
\varphi \frac{\partial S_{0}}{\partial t}=-\frac{\partial u_{o}}{\partial x} \\
\varphi \frac{\partial S_{g}}{\partial t}=-\frac{\partial u_{g}}{\partial x}+q_{g}
\end{gathered}
$$

where $q_{s}$ is the source term at the injection block.

The saturations of the phases are related by means of the following restriction equation.

$$
S_{o}+S_{g}=1
$$

The capillary pressure is defined as:

$$
P_{c o g}=P_{g}-P_{o}
$$

Upon combination and rearrangement of Equations (15) to (20), gives the general form of pressure and saturation equations.

$$
\begin{gathered}
\frac{\partial}{\partial x}\left[\frac{k k_{r o}}{\mu_{o}}\left(\frac{\partial P_{o}}{\partial x}-\gamma_{o} \frac{\partial z}{\partial x}\right)\right]=\varphi\left(\frac{\partial\left(1-S_{g}\right)}{\partial t}\right) \\
\frac{\partial}{\partial x}\left[\frac{k k_{r g}}{\mu_{g}}\left(\frac{\partial P_{o}}{\partial x}+\frac{\partial P_{c o g}}{\partial x}-\gamma_{g} \frac{\partial z}{\partial x}\right)\right]=\varphi\left(\frac{\partial\left(S_{g}\right)}{\partial t}\right)+q_{g}
\end{gathered}
$$

Equations (21) and (22) are solved subject to the following initial and boundary equations:

\section{Initial condition}

The core is initially assumed to fully saturate with oil

$$
\text { @ } t=0 \quad S_{g}=0
$$

In addition, the initial oil phase pressure distribution is obtained from hydrostatic equilibrium by assuming atmospheric pressure at the inlet face (top of the core).

\section{Inlet-face boundary condition}

Constant gas injection rate into the injection face is accounted for in the simulation. This is done by considering this face as a no flow boundary (zero oil velocity) and a source term for the gas injected into the corresponding block.

$$
\text { @ } Z=0 \quad u_{o}=0
$$

In the corresponding block:

$$
q_{g}=q_{i n j}
$$

\section{Outlet-face boundary condition}

Capillary end effect at the outlet imposes a zero capillary pressure and outlet pressure is constant atmospheric pressure.

$$
\begin{gathered}
\text { (a) } Z=L \quad P=14.7 \mathrm{psi} \\
\text { @ } Z=L \quad P_{\text {cog }}=0
\end{gathered}
$$

\subsubsection{Numerical Method}

The in-house numerical model has been developed in this study. The IMplicit-Pressure Explicit-Saturation method (IMPES) is used to solve the flow equations of oil and gas. A second-order centered finite-difference scheme is used to discretize the derivative terms. Single-point upwinding is used for the relative permeability terms to ensure numerical stability. Typically, 61 grid blocks are performed to accurately resolve saturation and pressure profiles within a model.

\subsubsection{History Matching}

The in-house developed numerical model has an ability to find the unknown parameters by history matching. History 
matching was performed by defining the following objective function to show the agreement between experimental and simulation oil production data.

$$
E=\sum_{i} \frac{\left(N_{p s m_{i}}-N_{p \exp }\right)^{2}}{N_{p \exp _{i}}^{2}}
$$

In which $i$ represents a time point and $\sum$ is over an enough number of data points that allows a smooth curve to fit the data points using cubic spline method. This objective function is minimized if first derivative of the objective function is equal to zero. For example, if three parameters $\left(e . g . n_{o}, n_{g}\right.$ and $s_{\text {org }}$ ) should be determined by matching the simulated and experimental results, $E$ must be minimized. Therefore, the following system of equations should be solved:

$$
\begin{aligned}
& \frac{\partial E_{\left(n_{o}, n_{g}, s_{\text {org }}\right)}}{\partial n_{o}}=0 \\
& \frac{\partial E_{\left(n_{o}, n_{g}, s_{\text {org }}\right)}}{\partial n_{g}}=0 \\
& \frac{\partial E_{\left(n_{o}, n_{g}, s_{\text {org }}\right)}}{\partial s_{\text {org }}}=0
\end{aligned}
$$

In this system, derivatives must be calculated numerically using Newton's method. The elements of the Jacobin matrix should be calculated numerically (Broyden, 1965). The Broyden method is used for updating the Jacobin matrix to minimize the number of function evaluations. Any number of matching parameters can be determined using this approach, but increasing the number of unknowns clearly increases the non-uniqueness of the results.

\section{RESULTS}

The experimental studies were categorized into two main sections. One category consists of what we shall call "gravity dominated" experiments where a high permeability porous medium and $120 \mathrm{~cm}$ long core holder was used. Under this condition the capillary threshold height plays a small role. The second category includes "capillary dominated experiments" where permeability was lower and a $60 \mathrm{~cm}$ core holder was used. As we shall see, in these tests the residual oil saturation and shape of relative permeability curves should be corrected against capillary end-effect.

Different gas injection rates are used for each type of porous medium to determine the influence of displacement instability on gas/oil relative permeability and residual saturation. Table 1 gives the key parameters for these experiments. In this table, the porous medium with similar texture, porosity and permeability have been identified with different letters; the "a", "b" and "c" sands exhibit permeability values of approximately 18, 9 and 3.5 Darcy. In each pair, the comparison is performed in configurations where the viscous forces and gravity compete.

\subsection{Gravity Dominated Experiments}

In this case, displacement rate is changed from gravity to viscous dominated regions, where the capillary end-effect is negligible. For these tests, the gas relative permeability is determined by history matching technique, when the oil relative permeability and residual saturation are known from the analytical procedure and experimental data respectively. Same procedure as explained in Section 2.1.3 is used to calculate the relative permeability for all experiments conducted in this category. Figure 4 shows the effect of displacement
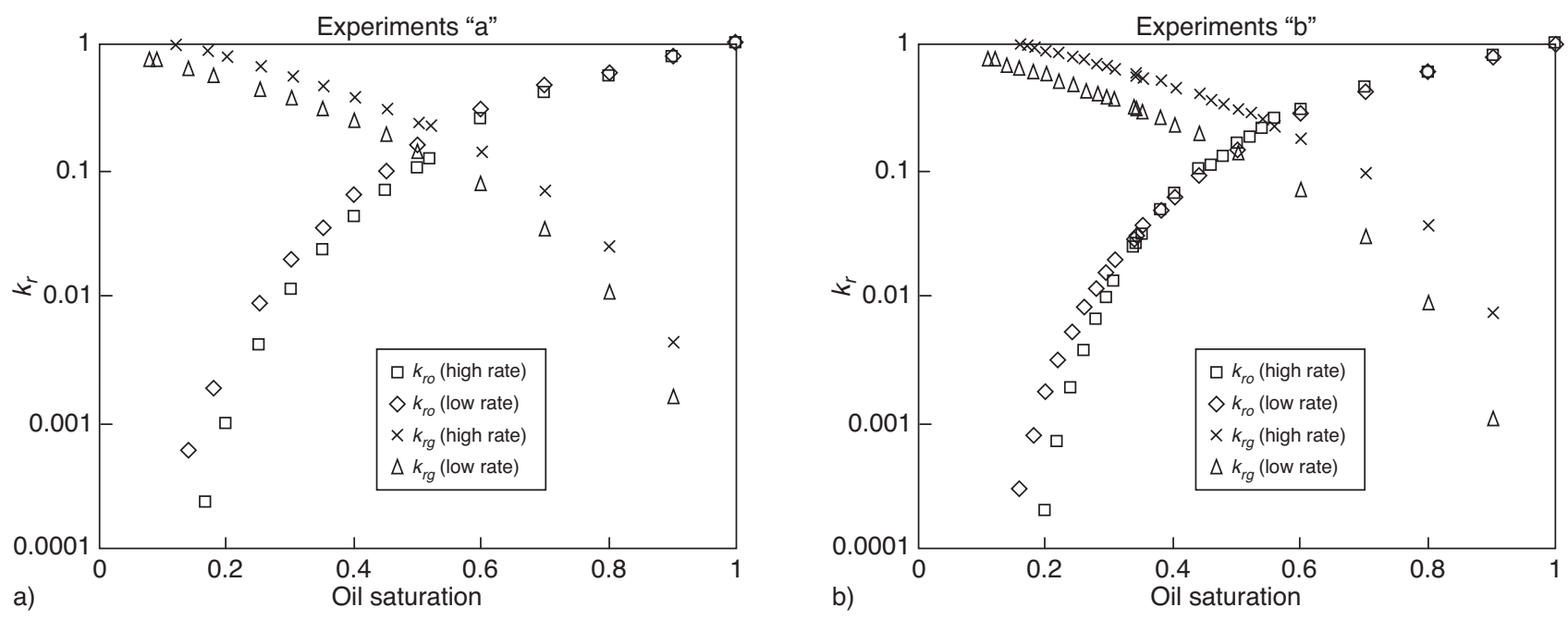

Figure 4

Comparison of gas/oil relative permeability under various displacement rate: a) Experiments "a"; b) Experiments "b". 


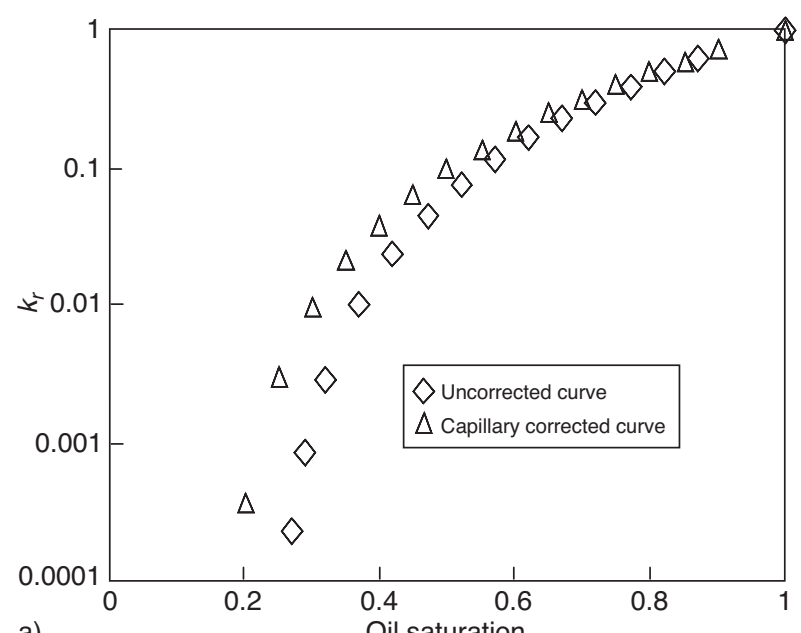

a)

Figure 5

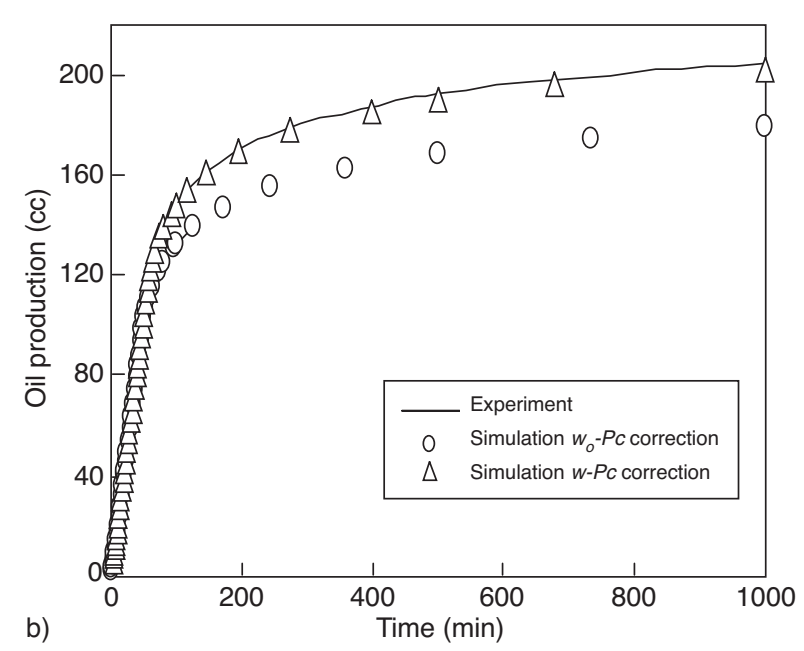

b)

When capillary effect is important: a) Oil relative permeability before and after capillary end-effect correction;

b) Oil production history matching with and without capillary end-effect correction.

velocity on two-phase relative permeability for experiments "a" and "b". Each sub-figure presents the relative permeability curves for the highest and lowest values of the displacement rate.

For the high displacement velocity experiments (normally greater than the stabilized velocity) the early time production data is found to be sensitive to the gas relative permeability. This behavior is enhanced by increasing the flow rate. As expected, for the low gas displacement velocity around the critical velocity, the oil relative permeability is slightly higher. This difference is mostly apparent at the low oil saturations, where the film flow controls the recovery mechanism. As the injection velocity increases, above the critical velocity, the invasion front becomes more unstable and the amount of bypassed oil increases. Hence, the residual oil saturation can be large. The relative permeability to gas is also changed to the higher values by increasing the injection rate. This is more apparent for the high oil saturations that include the early time data.

\subsection{Capillary Dominated Experiments}

In this category of experiments, the porous medium length is half of the previous model and the permeability is significantly reduced by using the fine sand particles. Calculation of the capillary end-effect number suggests that the residual oil saturation and shape of relative permeability curve should be corrected against capillary end-effect. The retention of oil at the bottom of the porous medium causes the estimated remaining oil saturation to be higher than the residual oil saturation. After determination of relative permeability using remaining oil saturation from the analytical procedure, the

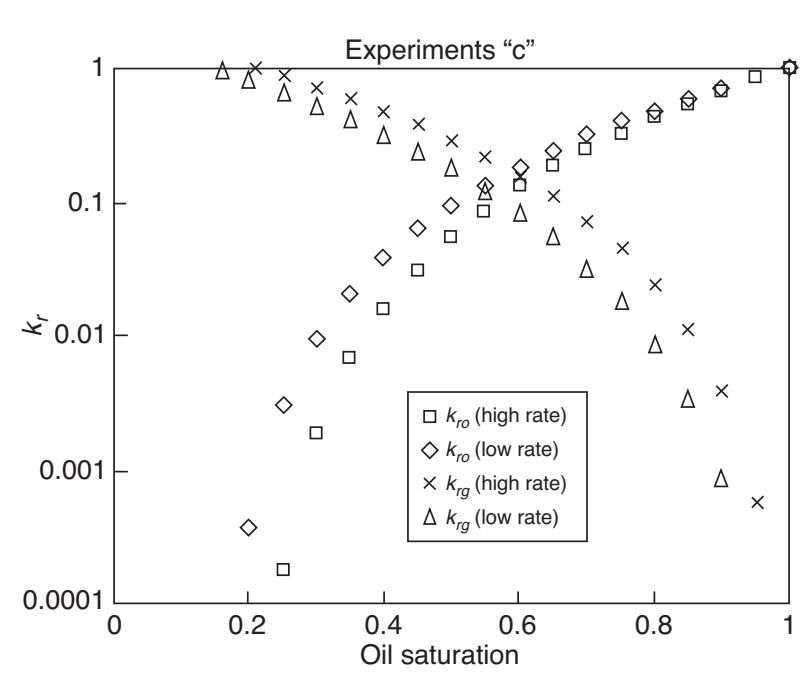

Figure 6

Comparison of gas/oil relative permeability under various displacement rate (Experiments "c").

history matching technique provided capillary corrected relative permeability and residual oil saturation. The known capillary pressure curve also needed as an input data.

The oil relative permeability computed before (derived by modified Hagoort's method) and after the capillary endeffect correction is compared in Figure 5a. A result of such a history matching is shown in Figure 5b. A comparison between relative permeabilities for stabilized and destabilized front velocity, when capillary effect is significant, is shown in Figure 6. 
As the flow rate increases, when the capillary hold-up zone is significant, the oil recovery is expected to decrease due to the flood front instability, but it should also increase due to increase in capillary number (i.e. leading the partial displacement of the oil trapped by the capillary threshold). However the high displacement velocity may reduce the endeffect, this is compensated by the large liquid saturation behind the front. In this study, the remaining oil saturation increases (oil recovery decreased) when displacement velocity increases.

Experimental results in both cases indicate that the degree of change in relative permeability and residual saturations are influenced by the front instability (viscous fingering) and petrophysical properties (permeability, porosity and heterogeneities).

\section{DISCUSSION}

When gravity is stabilizing and larger than the viscous forces, the invasion of a non-wetting phase into a wetting liquid is stable. This phenomenon could be understood by using a combination of Darcy's law and a mapping to percolation theory (Lovoll et al., 2005). Moreover, in order to investigate the interplay of gravity, viscous and capillary forces on oil recovery mechanism and remaining oil saturation, experimental results are analyzed using proper dimensionless numbers.

\subsection{Displacement Instability (Viscous Fingering)}

Particularly, the instability due to the low (gas to oil) viscosity ratio and high capillary number is commonly defined as viscous fingering. In this section, factors that affect the stability of the front, by increasing the injection velocity, are investigated. For top-down drainage with high viscosity contrast between gas and oil, a number of investigations carried out in laboratories (i.e. Terwilliger et al., 1951; Hagoort, 1980) suggest the following criterion for the displacement under stabilized condition.

$$
v_{c}>v
$$

where $v_{c}$ is the critical gravity drainage velocity defined by Blackwell and Terry (1959) and Dumore (1964).

$$
v_{c}=\frac{k \Delta \rho g}{\Delta \mu}
$$

This criterion represents the situation in which the gravity force effects are overcame by increased magnitude of viscous forces. This definition is also in line with the theoretical argument based on percolation theory (Wilkinson, 1984; Stauffer and Aharony, 1991) in a stabilizing gradient. The theory predicts the following scaling law for the displacement understable condition (Xu and Yortsos, 1997; Meheust et al., 2002).

$$
w \approx\left(N_{B}-N_{c}\right)^{-(\alpha / 1+\alpha)}
$$

where $w$ is the invasion front width and is the correlation length exponent in percolation theory. Then, Meheust et al. (2002) and Lovell et al. (2005) described the generalized Bond number, $N_{B}^{*}=N_{B}-N_{c}$, and showed that when $N_{B}^{*} \rightarrow 0$, the front width $w$ in Equation (34) diverges, then proposed the following criterion for interface stabilization.

$$
N_{B}-N_{c}=N_{B}^{*}>0
$$

After substitution and simplifications, the following criterion is expected.

$$
\Delta \rho g k / \mu_{o}>v
$$
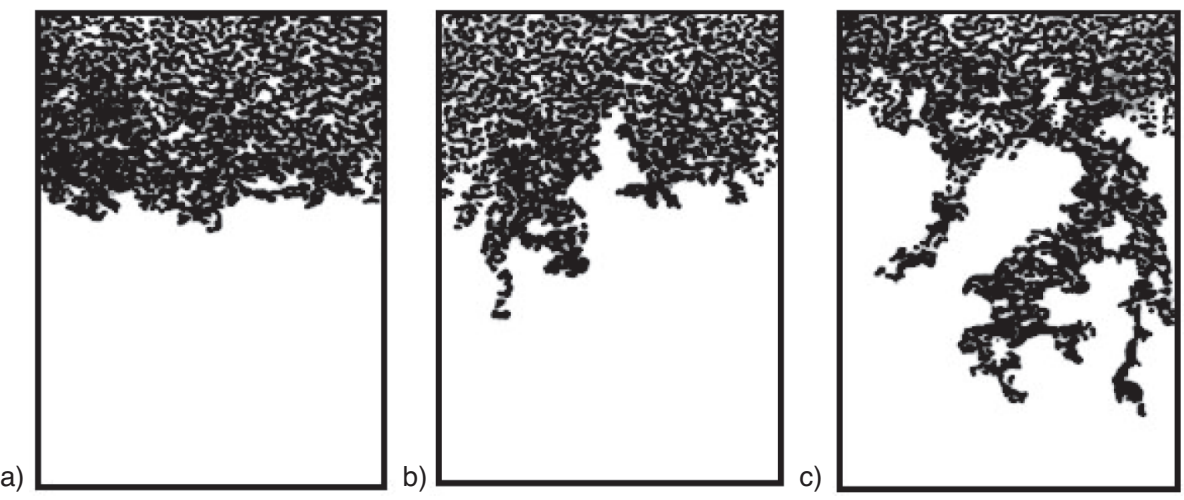

Figure 7

Front stability analysis from displacement structures for configurations: a) slow displacement; b) viscous fingering under gravity; c) high rapid flow. 
This relation is the same as Equation (32). The validity of the above criterion (Eq.32 or 36) can be confirmed using the visual observations of gas saturation shown in Figure 7, where parts a, b and c correspond to displacement velocities that are approximately $0.5,1.25$ and 2.6 times the critical velocity. The results in Figure 7 indicate that the displacement front has a stable form when the above criterion is met. In contrast, when the displacing velocity is exceeded than the critical velocity the displacement front becomes unstable.

\subsection{Dimensional Analysis}

Scaling of miscible and immiscible displacement in porous medium using dimensionless groups not only reduces the number of parameters to be studied, but also facilities in effectively capturing the multiphase mechanisms and fluid dynamics operative in these processes (Shook et al., 1992). In forced gravity drainage flow, the type of displacement observed during drainage depends on the relative magnitude of viscous forces and gravity, but also on their relative magnitude with respect to the heterogeneous capillary forces. In the following, we propose capillary number and Bond number to quantify the relative magnitudes of the prevailing forces.

In fluid dynamics, the capillary number represents the relative effect of viscous forces versus surface tension acting across an interface between a liquid and a gas, or between two immiscible liquids. It is defined as:

$$
N_{c}=\frac{\mu_{w} v}{\sigma \cos \theta}
$$

where $\mu_{w}$ is the wetting phase viscosity, $v$ is a characteristic velocity and $\sigma$ is the surface or interfacial tension between the two fluid phases. For low capillary numbers (a rule of thumb says less than $10^{-5}$ ), flow in porous medium is dominated by capillary forces.

The Bond number, $N_{B}$, is a dimensionless number expressing the ratio of body forces (often gravitational) to capillary forces.

$$
N_{B}=\frac{\Delta \rho g l^{2}}{\sigma}
$$

where $\Delta \rho$ is the density difference between two fluids, $g$ is the gravity acceleration in the direction of flow and $l$ is the characteristic length scale (often taken as the average grain radius, Morrow et al., 1988). In this study, we have used $(k / \varphi)$ instead of $\left(l^{2}\right)$.

In the following, the remaining oil saturation from each experiment at the specific time and pore volume injected are analyzed using the above dimensionless groups.

\subsubsection{Capillary Number}

In any gas injection process, the mobility ratio is typically unfavorable and the development of unstable fingers during gas displacement is imperative. The presence of viscous forces in a high-rate gas injection experiments, higher capillary numbers, may result in unstable invasion front and less hydraulic connectivity in oil phase.

Figure 8 shows the relationship between capillary number and remaining oil saturation for different porous medium (various Bond numbers). As it expected, increasing in capillary number has a negative effect on remaining oil saturation in forced gravity drainage flow, which results in poor sweep efficiency. The effect of capillary number is shown at relatively constant Bond number. It is apparent from Figure 8 that one specific correlation could not fit all experimental data.

\subsubsection{Bond Number}

Remaining oil saturation as a function of the Bond number is shown in Figure 9. Results indicate that recovery at a constant capillary number increases with Bond number. This behavior is line with our expectation about the effect of capillarity on oil drainage by gas injection when the oil is the wetting phase. As Bond number increases i.e., the importance of the capillary force with respect to the gravity force decreases, oil recovery improves. Again, a plot of remaining oil saturation from all forced gravity drainage experiments versus their Bond numbers seems to be misleading. In the other words, the Bond and capillary number alone is insufficient to describe the effect of prevailing forces on forced gravity drainage mechanism.

\subsubsection{Combined Group}

Examination of residual oil saturations as a function of dimensionless groups suggests that none of the above scaling groups individually can predict the remaining oil saturation. In the following, we suggest a combined group that could

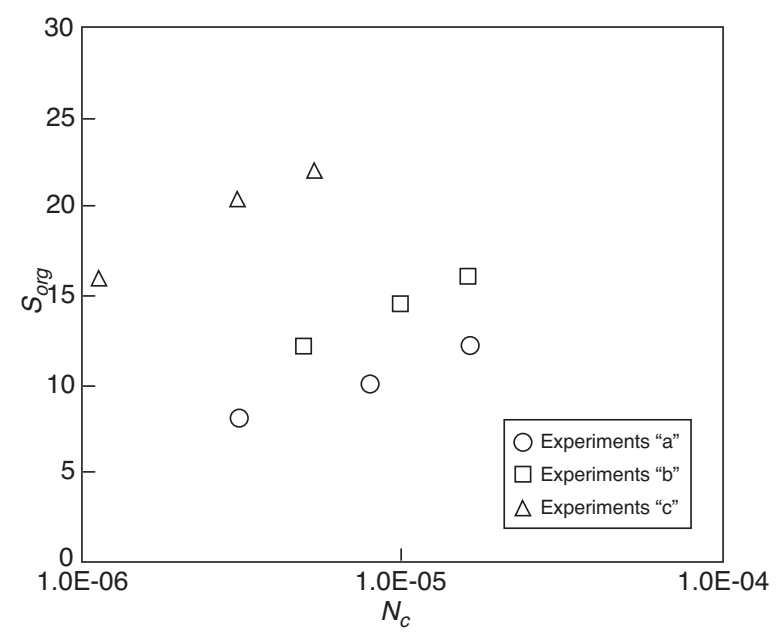

Figure 8

Final residual oil saturation from all experiments against capillary number. 


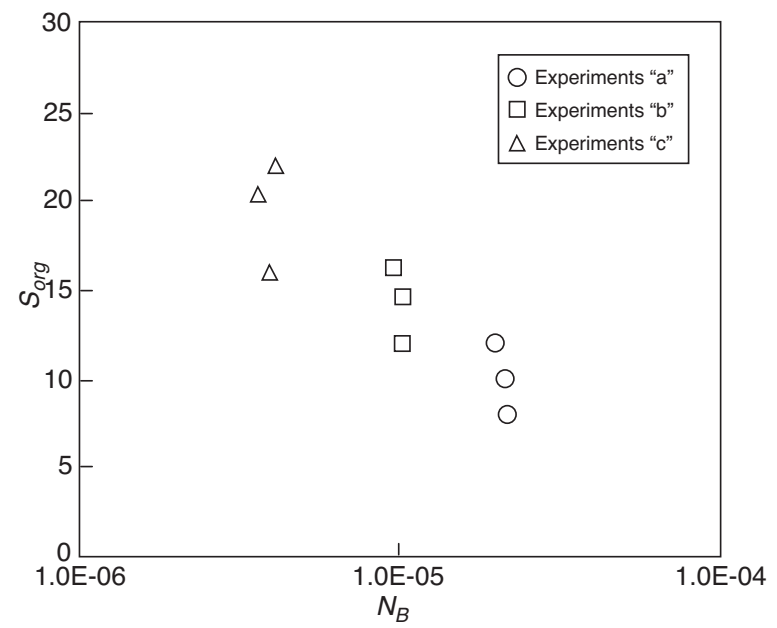

Figure 9

Final residual oil saturation from all experiments against Bond number.

capture the dependence of remaining oil saturation over the whole range of variables investigated. According to the experimental results shown in Figures 8 and 9, residual oil saturation has an opposite relation to the Bond and capillary number. It is also demonstrated that the effect of capillary number is less than the importance of Bond Number. Therefore, the existence of the scaling factor is necessary.

According to the above explanation, the following group is proposed.

$$
N_{C o}=\frac{N_{B}}{N_{c}^{A}}
$$

where $A$ is the scaling factor. The results are shown in Figure 10, in which the remaining oil saturations at breakthrough time, after 2 pore volumes injection and at the end of each experiment are plotted against $N_{C o}$ for all experiments. The slope of the correlation and the value of the scaling factor changed when the remaining oil saturation at different period of production is plotted. The plot of remaining oil saturation at breakthrough time shows more negative slope and the scaling factor has a larger value. This indicates that the breakthrough time recovery is more affected by change in capillary number (destabilized velocity). As the time passes and the process advances, dependency of the internal fluid distribution on capillary number reduces.

This dimensionless group successfully combines the effect of main forces exist in forced gravity drainage flow. The results suggest that within the range of experiments conducted here, there is a logarithmic relationship between the residual saturation and proposed group.

$$
S_{\text {org }}=C\left(\operatorname{Ln}\left(N_{B}\right)-A \operatorname{Ln}\left(N_{c}\right)\right)+D
$$

where $C$ and $D$ are constants.
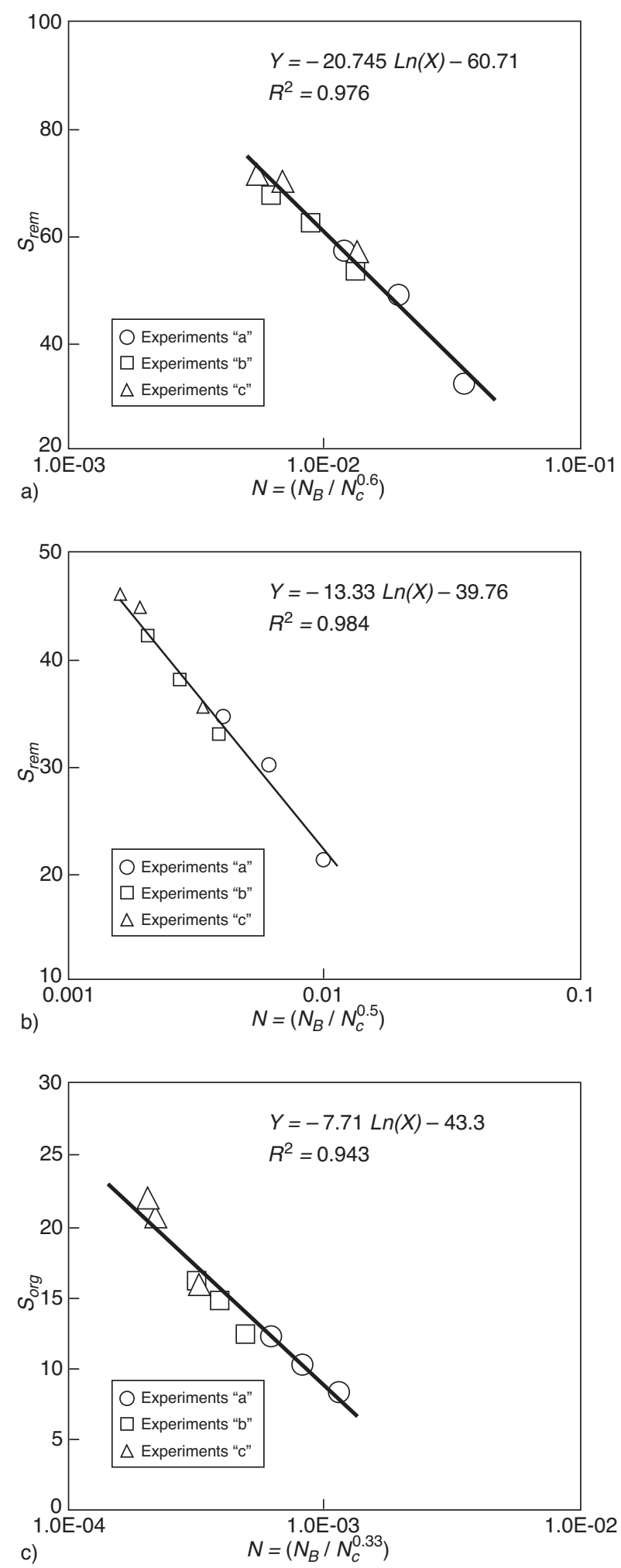

Figure 10

Residual oil saturation from all experiments versus the new dimensionless group: a) remaining oil saturation at breakthrough; b) remaining oil saturation after $2 \mathrm{PV}$ injection; c) final residual oil saturation. 
Applicability of the proposed correlation beyond the range of conducted experiments needs to be further examined, especially when the value of the proposed dimensionless group is significantly reduced below values investigated in this study.

\section{CONCLUSIONS}

Forced gravity drainage experiments of the immiscible displacement of a wetting phase (synthetic oil) by a nonwetting phase (Nitrogen) are performed on a synthetic porous medium (bead-pack and sand-pack), under constant viscosity ratio and varying values of capillary number (displacement rate) and Bond Number (Permeability). Analytical and numerical models are used to estimate relative permeabilities. The analytical model used the fundamental of the Hagoort's formulation to calculate the oil relative permeability. The numerical model consists of the conventional equations describing the 1-D macroscopic flow of two fluids in an isotropic and homogenous porous medium. Structural analysis of the fluid distribution in porous medium allows analyzing the stability of the invasion front by gradient percolation theory. The experimental result also analyzed using dimensionless numbers and new group proposed to describe the recovery mechanism under forced gravity drainage flow. According to the experimental studies and the mathematical analysis presented in this paper, the most important conclusions are outlined below:

- The estimated relative permeability and residual saturation are sensitive to the dominant flow regimes, which changes gradually from the gravity stabilize to the viscous drive. The oil relative permeability is higher in the gravity dominated case, whereas the gas relative permeability and residual oil saturation are higher when the viscous forces dominate;

- The high oil saturations are mostly affected by gas relative permeability, whereas the relative permeability to oil is mostly pronounced at the low oil saturations where the film flow controls the recovery mechanism;

- Scaling analysis using dimensionless numbers indicates that residual oil saturation decreases with increased Bond number and increases with increased capillary number. Furthermore, a proposed dimensionless group combining the effect of gravity and viscous forces to capillary force shows good agreement for prediction of remaining oil saturations for all experiments conducted in this study.

\section{ACKNOWLEDGMENTS}

The authors gratefully acknowledge Dr. M. Pooladi-Darvish for his unconditional availability for technical comments during this study. Acknowledge is extended to the laboratory assistance of PUT research center, H. Salimi, for his kindly assistant during laboratory working. We would also like to thank Mrs. M. Khosravi for her assistant during the preparation of this manuscript. Authors acknowledge is also extended to the Iranian Central Oil Field Company (ICOFC) for the financial support.

\section{REFERENCES}

Ali J.K. (1997) Development in measurement and interpretation techniques in core flood tests to determine relative permeabilities, SPE paper 39016, Rio de Janeiro, Brazil, 29 August-2 September.

Avraam D.G., Payatakes A.C. (1995) Flow Regimes and Relative Permeabilities during Steady-State Two-Phase Flow in Porous Media, J. Fluid Mech. 293, 207.

Birovljev A., Furuberg L., Feder J., Jossang T., Maloy K.J., Aharony A. (1991) Gravity invasion percolation in two dimensions: experiment and simulation, Phys. Rev. Lett. 67, 584-587.

Blackwell J.T., Terry M.W. (1959) Factors influencing the efficiency of miscible displacement, Trans AIME 216, 1-8.

Blunt M., King P. (1991) Relative permeabilities from two- and three-dimensional pore-scale network modeling, Transport Porous Med. 6, 407-433.

Broyden C.G. (1965) A Class of Methods for Solving Nonlinear Simultaneous Equations, Math. Comput. 19, 92, 577-593.

Constantinides G.N., Payatakes A.C. (1996) Network Simulation of Steady-State Two-Phase Flow in Consolidated Porous Media, AIChE J. 42, 369.

Dumore J.M. (1964) Stability consideration in downward miscible displacement, SPEJ 356-362.

Ferer M., Sams W.N., Geisbrecht R.A., Smith D.H. (1995) Fractal nature of viscous fingering in two-dimensional pore level models, AIChE J. 41, 749-763.

Ferer M., Bromhal G.S., Smith D.H. (2003) Pore-level modeling of immiscible drainage: validation in the invasion percolation and DLA limits, Physica A 319, 11-35.

Firoozabadi A., Aziz K. (1988) Relative permeability from centrifuge data, Proceeding of the 56th California regional meeting of the SPE, Okland, CA, 2-4 April, SPE paper 15059.

Fourar M., Bories S., Lenormand R., Persoff P. (1993) TwoPhase Flow in Smooth and Rough Fractures: Measurement and Correlation by Porous-Medium and Pipe Flow Models, Water Resour. Res. 29, 3699.

Goode P.A., Ramakrishnan T.S. (1993) Momentum Transfer across Fluid-Fluid Interfaces in Porous Media: a Network Model, AIChE J. 39, 1124-1993.

Gouyet J.F., Rosso M., Sapoval B. (1988) Fractal structure of diffusion and invasion fronts in three- dimensional lattices through the gradient percolation approach, Phys. Rev. B 37, 1832-1838.

Hagoort J. (1980) Oil recovery by gravity drainage, SPE J. 139150 .

Hirasaki G.J., Rohan J.H., Dudley J.W. (1995) Interpretation of oil-water relative permeabilities from centrifuge experiments, SPE Adv. Technol. 3, 1, 66-75.

Hughes R.G., Blunt M.J. (2000) Pore Scale Modeling of Rate Effects in Imbibition, Transport Porous Med. 40, 295.

Jones S.C., Roszelle W.O. (1978) Graphical techniques for determining relative permeability from displacement experiments, J. Petrol. Sci. Tech. 30,807-817.

Lovoll G., Meheust Y., Maloy K.J., Aker E. (2005) Competition of gravity, capillary and viscous forces during drainage in a two- 
dimensional porous medium, a pore scale study, Energy J. 30, 6, 861-872.

Meheust Y., Lovoll G., Maloy K.J., Schmittbuhl J. (2002) Interface scaling in a $2 \mathrm{~d}$ porous medium under combined viscous, gravity and capillary effects, Phys. Rev. E 66, 51603-51615.

Mohanty K.K., Miller A.E. (1991) Factors influencing unsteady relative permeability of a mixed-wet reservoir rock, Soc. Petrol. Eng. Form. Eval. 6, 349-358.

Or D. (2008) Scaling of capillary, gravity and viscous forces affecting flow morphology in undersaturated porous media, $A d v$. Water Resour. 31, 1129-1136.

Persoff P., Pruess K. (1995) Two-Phase Flow Visualization and Relative Permeability Measurement in Natural Rough-Walled Rock Fractures, Water Resour. Res. 31, 1175-1995.

Philip J.R. (1975) Stability analysis of infiltration, Soil Sci. Soc. Am. Proc. 39, 1042-1049.

Raats P.A.C. (1973) Unstable wetting fronts in uniform and nonuniform soils, Soil Sci. Soc. 36, 681-685.

Saeedi M. (2007) Modeling and experiments of drainage relative permeability and capillary pressure functions using a centrifuge, Msc thesis, University of Calgary, Canada, 2007.

Singh M., Mani V., Honarpour M.M., Mohanty K.K. (2001) Comparison of viscous and gravity dominated gas-oil relative permeabilities, J.Petrol.Sci.Eng. 30,67-81.

Singh M., Mohanty K.K. (2003) Dynamic Modeling of Drainage through Three-Dimensional Porous Materials, Chem. Eng. Sci. $\mathbf{5 8}, 1,3$

Skauge A., Haskjold G., Thorsen T., Aarra M. (1997) Accuracy of gas-oil relative permeability from two-phase flow experiments. SCA 9707, International Symposium of the Society of Core Analysis, Calgary, Canada.
Theodoropoulou M.A., Sygouni V., Karoutsos V., Tsakiroglou C.D. (2008) Relative permeability and capillary pressure functions of porous media as related to the displacement growth pattern, Int. J. Multiphas. Flow 31, 1155-1180.

Tsakiroglou C.D., Theodoropoulou M., Karoutsos V. (2003) Non-equilibrium capillary pressure and relative permeability curves of porous media, AIChE J. 49, 2472-2486.

Tsakiroglou C.D., Theodoropoulou M.A., Karoutsos V., Papanicolaou D. (2005) Determination of the effective transport coefficients of pore networks from transient immiscible and miscible displacement experiments, Water Resour. Res. J. 41, 2, W02014.

Virnovskey G.A., Skjaevaland S.M., Surdal J., Ingsoy P. (1995) Steady-state relative permeability measurements corrected form capillary effect. SPE 30541, Proceeding of the SPE annual technical conference and exhibition, Dallas, TX, 22-25 October.

Vizika O., Avraam D.G., Payatakes A.C. (1994) On the Role of the Viscosity Ratio during Low-Capillary Number Forced Imbibition in Porous Media, J. Colloid Interf. Sci. 165, 386.

Wilkinson D. (1986) Percolation effects in immiscible displacement, Phys. Rev. A 34, 1380-1391.

Xu B., Salin D., Yortsos Y.C. (1998) Invasion percolation with viscous forces, Phys. Rev. E 57, 739-751.

Zhang J.H., Liu Z.H. (1998) Study of the relationship between fractal dimension and viscosity ratio for viscous fingering with a modified DLA model, J. Petrol. Sci. Eng. 21, 123-128.

Zhang Y., Shariati M., Yortsos Y.C. (2000) The spreading of immiscible fluids in porous media under the influence of gravity, Transport Porous Med. 38, 117-140.

Final manuscript received in May 2009 Published online in November 2009 